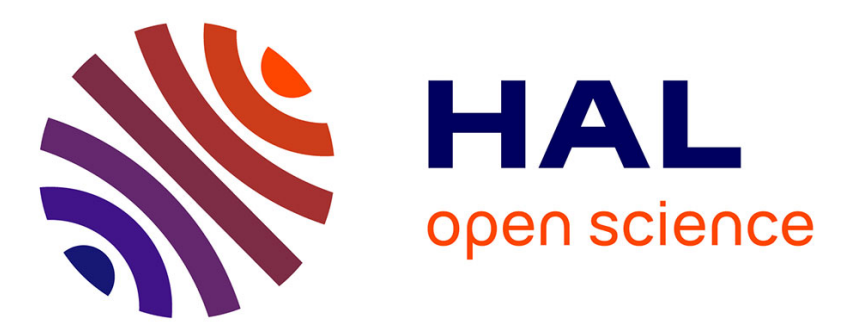

\title{
Automatic Characteristic Frequency Association and All-Sideband Demodulation for Detection of a Bearing Fault of a Test Rig
}

Marcin Firla, Zhong-Yang Li, Nadine Martin, Christian Pachaud, Tomasz Barszcz

\section{To cite this version:}

Marcin Firla, Zhong-Yang Li, Nadine Martin, Christian Pachaud, Tomasz Barszcz. Automatic Characteristic Frequency Association and All-Sideband Demodulation for Detection of a Bearing Fault of a Test Rig. Mechanical Systems and Signal Processing, 2016, 80, pp.335-348. 10.1016/j.ymssp.2016.04.036 . hal-01314866

\author{
HAL Id: hal-01314866 \\ https://hal.science/hal-01314866
}

Submitted on 12 May 2016

HAL is a multi-disciplinary open access archive for the deposit and dissemination of scientific research documents, whether they are published or not. The documents may come from teaching and research institutions in France or abroad, or from public or private research centers.
L'archive ouverte pluridisciplinaire HAL, est destinée au dépôt et à la diffusion de documents scientifiques de niveau recherche, publiés ou non, émanant des établissements d'enseignement et de recherche français ou étrangers, des laboratoires publics ou privés. 


\title{
Automatic Characteristic Frequency Association and All-Sideband Demodulation for Detection of a Bearing Fault of a Test Rig
}

\author{
Marcin Firla ${ }^{\mathrm{a}, *}$, Zhong-Yang $\mathrm{Li}^{\mathrm{a}}$, Nadine Martin ${ }^{\mathrm{a}, \mathrm{b}}$, Christian Pachaud ${ }^{\mathrm{c}}$, \\ Tomasz Barszcz ${ }^{\mathrm{d}}$ \\ ${ }^{a}$ Univ. Grenoble Alpes, GIPSA-Lab, F-38000 Grenoble, France \\ ${ }^{b}$ CNRS, GIPSA-Lab, F-38000 Grenoble, France \\ c29, Rue Antoine Condorcet, 38090 Villefontaine, France \\ ${ }^{d}$ AGH University of Science and Technology, Faculty of Mechanical Engineering and \\ Robotics, al. Mickiewicza 30, 30-059 Kraków, Poland
}

\begin{abstract}
This paper proposes advanced signal-processing techniques to improve condition monitoring of operating machines. The proposed methods use the results of a blind spectrum interpretation that includes harmonic and sideband series detection. The first contribution of this study is an algorithm for automatic association of harmonic and sideband series to characteristic fault frequencies according to a kinematic configuration. The approach proposed has the advantage of taking into account a possible slip of the rolling-element bearings. In the second part, we propose a full-band demodulation process from all sidebands that are relevant to the spectral estimation. To do so, a multi-rate filtering process in an iterative schema provides satisfying precision and stability over the targeted demodulation band, even for unsymmetrical and extremely narrow bands. After synchronous averaging, the filtered signal is demodulated for calculation of the amplitude and frequency modulation functions, and then any features that indicate faults. Finally, the proposed algorithms are validated on vibration signals measured on a test rig that was designed as part of the European Innovation Project 'KAStrion'. This rig simulates a wind turbine drive
\end{abstract}

\footnotetext{
* Corresponding author

Email address: marcin.firla@gipsa-lab.grenoble-inp.fr (Marcin Firla)
} 
train at a smaller scale. The data show the robustness of the method for localizing and extracting a fault on the main bearing. The evolution of the proposed features is a good indicator of the fault severity.

Keywords: signal processing, condition monitoring, characteristic fault frequencies, kinematics, amplitude demodulation, frequency demodulation

\section{Introduction}

Condition monitoring systems (CMSs) are widely used in industry and can provide considerable benefits for surveillance of mechanical components [1, 2]. CMSs are based on data acquisition and processing that is designed to reveal 5 abnormalities in the state of the investigated system. There are several techniques to perform this task, such as vibration, acoustic emission, and lubricant analysis. This study focuses on vibration-based condition monitoring, which is the key component of predictive maintenance as it can provide indicators that are related to the evolution of potential faults and failures $[3,4]$.

The development of CMSs is an active research domain $[5,6,7,8,9,10]$. Each branch of industry where there are crucial and expensive rotating components used, as in the oil industry during the extraction of oil, or numerous and spread systems, as in the wind industry, could benefit from CMSs to monitor the state of mechanical components.

Early detection of mechanical faults requires automatic interpretation of a large amount of data for the surveillance to be feasible [11]. Mechanical faults in rotating machines, and particularly those associated with rolling-element bearings, create modulations in the vibration signals. These modulations are visible on the spectrum as sidebands. Demodulation of these sidebands can provide

20 features that are indicative of the developing faults. This demodulation is a widely-used and well-proven technique in the diagnosis of vibration signals $[12,13,14,15]$. The diagnostics of bearing faults using demodulation analysis is an active research area. Recent contributions include the 'max-med' estimator 
for the optimal selection of the carrier frequency [16], and envelope analysis of 25 speed transients [17].

This paper proposes an automatic fault detection and diagnosis method in a fully automatic tool, known as 'AStrion'. This method consists of several steps for the detection of the most common drive-train faults, like gear and bearing failures. The tool is based on sophisticated data validation and time-frequency representation analysis. AStrion is particularly efficient in the processing of stationary signals with a rich spectral composition.

In this paper, the spectrum analysis is based on an automatic peak detection and classification method $[18,19,20]$ that provides a data-driven approach without any a-priori information on the data. The harmonic series and the

35 sidebands around each harmonic are then identified from the peaks according to their frequencies and classes [21]. On the basis of the data from this sideband identification, this paper proposes two further new algorithms. The first is a kinematic association method that is used to link the harmonics directly to the mechanical components. It is an exhaustive algorithm that takes into account all of the information available from the signal, and all of the possible combinations of modulations that can arise from the kinematic configuration of the system. The core algorithm is a data-driven method for the selection of one harmonic series among numerous proximate ones. This automatic process features within a new method for the selection of rolling-element bearing frequencies. The sec-

45 ond of these new algorithms is a sideband demodulation technique that is used to calculate the features directly from the modulation created by a mechanical fault. By taking advantage of the previously identified modulation sidebands, the demodulation can be repeatedly applied to all of the identified sidebands, to investigate the entire frequency span. Such a comprehensive approach has so never been reported previously. Finally, new fault indicators are proposed that are based on the estimated amplitude and frequency modulation functions.

The proposed fault indicators have been compared against commonly used indicators, including the crest factor, kurtosis, and root mean square (RMS) ones. As [22] indicated, the listed indicators are very often used for predictive 
maintenance strategies, and are not sufficient for this task. To facilitate advances in predictive maintenance, the handling of other indicators is needed, and this paper offers a set of advanced features for this purpose. Moreover, the proposed approach is also compared with a narrow-band RMS approach.

The paper is organized as follows. Section 2 presents the automatic characteristic fault frequency associations, and section 3 describes an original approach to demodulation. Section 4 presents the results of the methods applied to realworld data, and a comparison with traditional diagnostic approaches. Section 5 draws the conclusions and indicates the future perspectives.

\section{Characteristic Fault Frequency Associations}

65 Characteristic fault frequencies arise from the geometry of the system investigated, and they can be used to help to find and identify the origin of a fault. These frequencies are easy to identify for components such as gears, shafts, and rolling-element bearings, which are important parts of the rotating machinery. When the kinematic information is known, the proposed method gives additional value to the AStrion methodology by highlighting the vibration patterns that can arise from the mechanical configuration of the system. However, AStrion can also operate without any information relating to the investigated system, and can perform all of the other analyses.

This section proposes a method to automatically associate characteristic

75 fault frequencies with the harmonic series and modulation sideband series that were previously detected using the algorithm proposed by [21]. These data are then used as the input for the proposed association method. In this context, the association is made critical as more than one series is often close to the theoretical value of the fault frequency. This issue has to be overcome.

\section{so 2.1. Characteristic Fault Frequencies}

The well-known characteristic fault frequencies in rotating machinery depend on the geometry and speed of the components. The commonly used formulae 
Table 1: Selected formulae for the calculation of characteristic fault frequencies [23].

\begin{tabular}{|l|l|}
\hline Fault frequency name & Formula \\
\hline Shaft speed frequency & $S S F=V_{r}$ \\
Gear mesh frequency & $G M F=V_{r} \cdot z$ \\
Ball-pass frequency of outer race & $B P F O=\frac{n_{r} V_{r}}{2}\left\{1-\frac{d_{r}}{d_{p}} \cos (\phi)\right\}$ \\
Ball-pass frequency of inner race & $B P F I=\frac{n_{r} V_{r}}{2}\left\{1+\frac{d_{r}}{d_{p}} \cos (\phi)\right\}$ \\
Fundamental train frequency & $F T F=\frac{V_{r}}{2}\left\{1-\frac{d_{r}}{d_{p}} \cos (\phi)\right\}$ \\
Ball spin frequency & $B S F=\frac{V_{r} \cdot d_{c r}}{2 d_{r}}\left\{1-\left(\frac{d_{r}}{d_{p}} \cos (\phi)\right)^{2}\right\}$ \\
\hline
\end{tabular}

are presented in Table 1 , where $z$ is the number of teeth in a gear, $V_{r}$ is the shaft speed, $n_{r}$ is the number of rolling elements, $d_{r}$ is the diameter of the rolling elements, $d_{p}$ is the pitch diameter, and $\phi$ is the angle of the load from the radial plane.

Usually, $V_{r}$ is measured only on one shaft, e.g. the high-speed shaft of a wind turbine. In such a situation, it is necessary to recalculate $V_{r}$ for each shaft in the system, which can be achieved using the speed ratio between each stage so of the gearbox.

Table 1 presents the formulae based on theoretical models for calculation of the characteristic frequencies of rotating machinery components [23]. These frequencies are referred to as the theoretical values. It is important to note that in the case of rolling-element bearings, the characteristic fault frequencies assume no slippage, whereas this must occur when a bearing is running. This will apply to the ball-pass frequency of the outer race $(B P F O)$, the ball-pass frequency of the inner race $(B P F I)$, the fundamental train frequency $(F T F)$, and the ball spin frequency $(B S F)$. This is why the estimated characteristic frequencies vary slightly when real-word applications are compared to theoretical values. Generally, it is normal to observe random variations within the range 
of $1 \%$ to $2 \%$ [24]. The next subsection presents the proposed method that is designed to overcome this effect.

\subsection{Proposed Method for the Association}

The proposed method for the use of kinematic information is a data-driven approach. This requires a-priori interpretation of the spectral content of a signal. This interpretation is provided by the AStrion steps, which include spectral component identification [19] and harmonic series and modulation sideband series detection [21]. The proposed algorithm associates the characteristic fault frequencies with the previously detected harmonic and sidebands series. Figure 1 shows a flowchart of the proposed method.

First, the characteristic fault frequencies have to be calculated. For this operation, the proposed method uses the rotational speed and the list of the theoretical characteristic fault frequencies expressed as orders. This list is computed according to the formulae of Table 1, although the rotational speed $V_{r}$ 115 is assumed to be $1 \mathrm{~Hz}$. This value is commonly used as a reference, as it can be directly applied to a signal after angular resampling, which is also known as order tracking. More details on the resampling can be found in [25, 23, 26]. Moreover, the speed can be measured only on one shaft in the system, provided that the list of frequencies as orders is recalculated with the use of gear ratios for a speed of each shaft that ensures that the speed of the shaft that carries a component is taken into account. This procedure is adapted for more complex systems composed of multiple shafts operating at a different rotational speed.

The association of the characteristic fault frequencies is then performed in two steps. First, the harmonic series that meets a criterion for the theoretical values are selected as possible candidates. These are followed by the final selection, which provides the association of a single harmonic series with one theoretical characteristic frequency. In a second step, the same set of operations is performed on the modulation sideband series. The difference with the harmonic series is that one characteristic fault frequency can be associated with a 


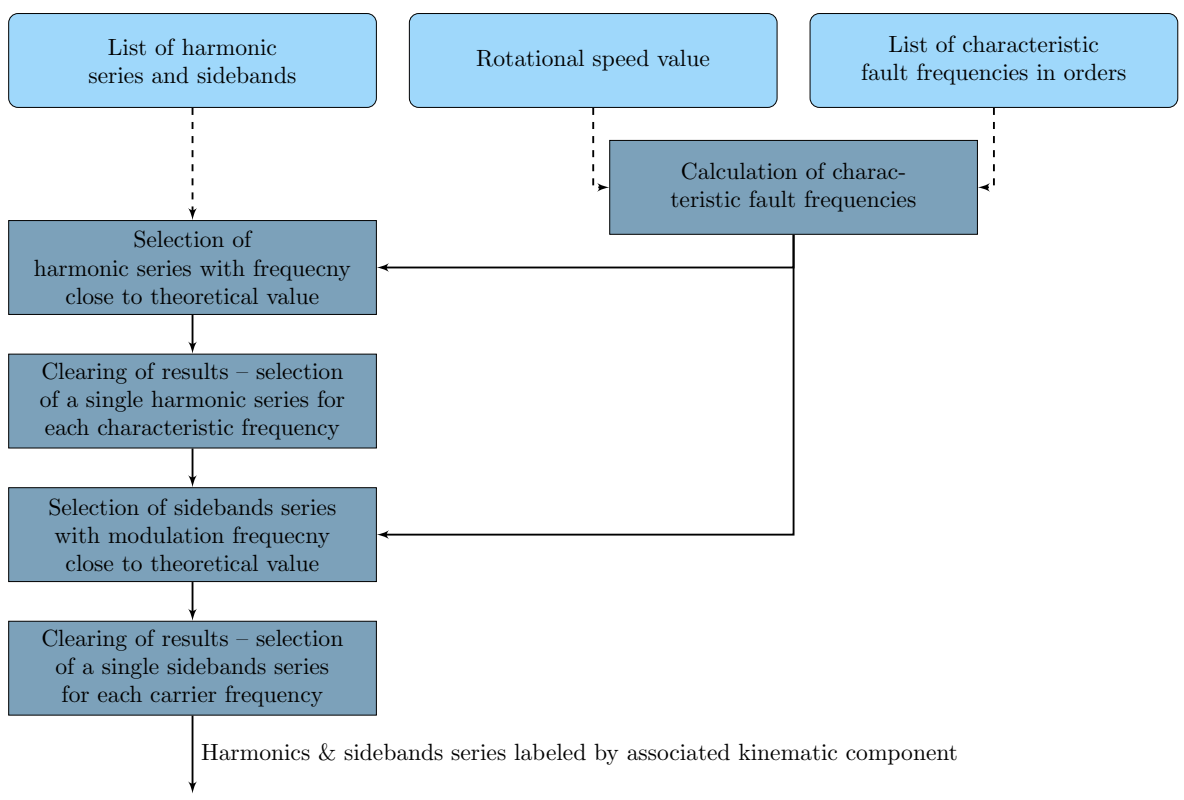

Figure 1: Flowchart of the characteristic fault frequency association algorithm.

single harmonic series, whereas it can be associated with numerous modulation series, which have a different carrier frequency.

The selection criterion is based on the relative difference between the theoretical and estimated values of the characteristic fault frequency, which is referred to as $R F D_{i}$

$$
R F D_{i}=\frac{f_{d, i}-f_{t, i}}{f_{t, i}} \cdot 100 \%,
$$

where $i$ is the index of a series, $f_{d, i}$ is the fundamental frequency of the estimated series, and $f_{t, i}$ is the theoretical frequency. The series with an absolute value of $R F D_{i}$ lower than a threshold $\eta$ are selected as potential candidates

$$
\left|R F D_{i}\right|<\eta .
$$

Moreover, due to the slippage phenomenon mentioned earlier, the threshold for the rolling-element bearings should be bigger than for all of the other mechanical components, and it is equal to $\eta+\delta \eta$. We propose to use $\eta$ and $\delta \eta$ as $1 \%$. 
The next step is to select only one series for the final association. The association of series with the characteristic fault frequencies corresponding to shafts and gearboxes is intuitive. The correct series is selected as the one with the lowest $R F D_{i}$.

In the case of rolling bearings, the lowest $R F D_{i}$ is not a sufficient indicator, because of the slippage phenomenon. Thus, an additional parameter is proposed, which is the bearing series indicator, which is defined as

$$
B S I_{i}=\frac{\frac{E_{i}}{E_{\max , i}}+\operatorname{den}_{i}}{2},
$$

where $E_{i}$ is the energy of the series under investigation, $E_{\max , i}$ is the energy of the series with the highest energy among the series selected in the first step, and $d e n_{i}$ is the density of the series. These features are computed using the algorithm used for harmonic series and sideband detection. The energy $E$ is computed as a square sum of the amplitudes of the peaks in the series, and the density of a series den describes the number of gaps in it, knowing that for a series without any gap $d e n=1$, which is the maximal value. For a detailed definition of these parameters, please refer to [21]. $B S I_{i}$ varies from 0 to 1 , where the highest value describes a series with the highest energy among the selected candidates and without gaps. Therefore, the series $i$ with the highest $B S I_{i}$ among the selected series in the first step is associated with the theoretical characteristic fault frequencies of a rolling-element bearing.

\section{Sideband Demodulation}

A number of methods exist for selecting the carrier frequency and spectral bands of a modulated signal $[16,27,24,28]$. The need for these techniques arises as for bearing diagnosis it is recommended to separately select the appropriate resonance frequency of a bearing for each investigated case. Contrary to the methodologies described in the literature, this paper proposes to perform the demodulation not just for one selected frequency band, but for the entire 
spectrum. As a result of the demodulation, we propose to calculate a list of sideband features, which can describe the system health.

A discrete signal $y[n]$ is considered as the sum of a band-limited deterministic part $s[n]$ and a wide-band random part $e[n]$

$$
y[n]=s[n]+e[n] .
$$

$s[n]$ can be expressed as

$$
s[n]=A[n] \cos (\Phi[n]),
$$

where $A[n]$ is the amplitude modulation, and $\Phi[n]$ is the instantaneous phase modulation.

The amplitude modulation can be written as

$$
A[n]=A_{0}\left(1-\alpha \cos \left(2 \pi f_{a} n\right)\right)
$$

where $A_{0}$ is the average amplitude, $\alpha$ is the amplitude modulation index, and $f_{a}$ is the frequency of the amplitude modulation function. The instantaneous phase $\Phi[n]$ and instantaneous frequency $F[n]$ modulation functions are written as

$$
\begin{aligned}
& \Phi[n]=2 \pi f_{0} n+\beta \sin \left(2 \pi f_{\Phi} n\right), \\
& F[n]=f_{0}+\beta f_{\Phi} \cos \left(2 \pi f_{\Phi} n\right) .
\end{aligned}
$$

where $f_{0}$ is the carrier frequency, which depends on the rotational speed and the resolution of the tachometer, $\beta$ is the frequency modulation index, and $f_{\Phi}$ is the frequency of the phase modulation function. Since the signal is modulated simultaneously in the amplitude and frequency, the Fourier series expansion s[n] of Equation (5) is

$$
s[n]=A_{0} \sum_{k=0}^{+\infty}\left(J_{k}(\beta)+\alpha J_{k \pm 1}(\beta)\right) \cos \left(2 \pi\left(f_{0} \pm k f_{\Phi}\right) n\right),
$$

where $k$ is the order of the sideband, $k=0$ corresponds to the carrier, and $J_{k}(\cdot)$ is the Jacobian polynomial of order $k$. $\beta$ is determined by

$$
\beta=\frac{f_{0}}{f_{\Phi}} \cdot r_{F M} \cdot \frac{2 \pi}{\Delta \phi},
$$


where $\frac{2 \pi}{\Delta \phi}$ is the tachometer resolution that specifies the number of tachometer samples per revolution, and $r_{F M}$ is the frequency modulation rate, which rarely

165 exceeds $10 \%$ for a rotating shaft. As the frequency modulation yields an infinite number of sidebands, the Bedrosian theorem is not respected. However, under the hypotheses [29] that

- the carrier frequency $f_{0}$ is significantly higher than the frequency modulation frequency $f_{\Phi}$

- the sideband power becomes negligible at the maximum frequency of $A[n]$;

the amplitude modulation $A[n]$ and the phase modulation $\Phi[n]$ can be recovered from the signal $s[n]$ using the Hilbert transform. The instantaneous frequency modulation $F[n]$ can be obtained from the phase signal $\Phi[n]$.

The demodulation algorithm can be decomposed into two steps. In the first step, the signal is band-pass filtered around the carrier frequency $f_{0}$, to isolate the spectral components that are not relevant to the phenomena to be analyzed. In the second step, the amplitude and frequency modulation functions are calculated after synchronous averaging. Hereinafter, we introduce the different steps of the algorithm.

Firstly, we propose to isolate the sidebands from the other spectral content using a multi-rate filtering method, described in Section 3.2. Then, as presented in Section 3.3, the filtered signal is processed with the time synchronous averaging to remove the information irrelevant to the modulation frequency. Afterwards the demodulation is performed, as explained in Section 3.4. Finally, some sideband features which characterize the amplitude and the frequency modulation functions are calculated from the averaged signal as shown in Section 3.5.

\subsection{Multi-Rate Filtering Technique}

The filtering method aims to preserve all of the spectral information within a frequency band $\mathbf{B}$

$$
\mathbf{B}=\left[f_{\text {inf }}, f_{\text {sup }}\right]
$$


where $f_{\text {inf }}$ and $f_{\text {sup }}$ are the lower and upper frequency boundaries of the filter, respectively, whereby both should be positive and below the Shannon frequency $190 \frac{F_{s y}}{2}$. The filter bandwidth is defined by $\Delta B=f_{\text {sup }}-f_{\text {inf } f}$.

The challenge comes if the bandwidth is very small or if the filter band is very close to the extreme values ( 0 and/or $\frac{F_{s_{y}}}{2}$ ). The design of a stable filter that satisfies the desired performance is difficult, as it is stable only at very low order. As a solution, and as originally used for reduction of the amount of data [30], use of a multi-rate filter inspired us to propose a new filtering technique that automatically decomposes a difficult filtering task into $N_{q}$ iterations, each of which is comprised of a chain of three essential operations, as described in subsection Methodology of the Multi-Rate Filter.

\section{Choice of the Filter Bandwidth}

On the Fourier spectrum of a signal expressed as in Equations (5) and (8), phase modulation will create an infinite number of sidebands at both sides of the carrier frequency $f_{0}$, which are spaced at an even interval $f_{\Phi}$. The energy of the high-order sideband is often hard to distinguish from the noise level, thus it is decided to demodulate only the frequency bandwidth which carries enough energy to identify the modulation sideband from the noise. The filter bandwidth is then selected based on the peak identification [19] and harmonic and sideband recognition [21] mentioned above. Therefore, the filter bandwidth can be chosen as

$$
\begin{array}{ll}
f_{\text {inf }}=f_{0}+K_{\text {inf }} \times f_{\Phi}, & K_{\text {inf }} \in \mathbb{Z}, K_{\text {inf }}<0, \\
f_{\text {sup }}=f_{0}+K_{\text {sup }} \times f_{\Phi}, & K_{\text {sup }} \in \mathbb{Z}, K_{\text {sup }}>0,
\end{array}
$$
of the sidebands. The filter bandwidth is proportional to $\left(\left|K_{\text {sup }}\right|-\left|K_{\text {inf }}\right|\right)$.

As no preliminary information is available on the modulation, filtering by empirically fixing $K_{\text {sup }}$ and $K_{\text {inf }}$ might distort the modulated signal, which may degrade the precision and is not the case for the proposed method. The is initially applied to identify all of the harmonic series and sidebands of the 
Table 2: Specification of the digital filters used in the multi-rate filtering

\begin{tabular}{|l||l|l|}
\hline Type & Butterworth & Elliptic \\
\hline Nature and operations involved & Low-pass \& high-pass & Band-pass \\
\hline Pass-band ripple & - & $0.005 \mathrm{~dB}$ \\
\hline Stop-band ripple & - & $-80 \mathrm{~dB}$ \\
\hline Stability criterion on the maximum of all of the poles & $<0.95$ & $<0.996$ \\
\hline Lowest order & 10 & 8 \\
\hline
\end{tabular}

spectrum, and to calculate the important parameters for the filter design, such as $f_{\Phi}, f_{0}, K_{\text {inf }}$, and $K_{\text {sup }}$, as long as the selected sidebands verify Carson's law [31]. Through this approach, all of the detectable sideband orders are taken 210 into account for the demodulation.

\section{Methodology of the Multi-Rate Filter}

The multi-rate filter consists of three basic operations: a frequency shift that applies a negative frequency shift $-\Delta f_{q}$ to the entire frequency contents; a filter that filters over the target filter band $\mathbf{B}_{q}$; and a down-sampling that decimates 215 the signal by a factor $D_{q}$.

For the filtering operation, a filter of sharp roll-off is required, and therefore the elliptic filter was selected. In the down-sampling operation and the frequency shifting step, a low-pass Butterworth filter is used to avoid the spectrum aliasing, considering its ripple-free feature. The specifications of the filters are presented in Table 2.

The algorithm selects the highest order of the filter that satisfies a stability criterion on the maximum of all of the poles. If the selected filter order is below the lowest order, the filter bandwidth is considered too narrow for the filter design. In this situation, the down-sampling and frequency shifting op225 erations are required. The details of the down-sampling rate selection and the frequency shift steps that are applied in the proposed filtering operation are given in Appendix A. 
The core operation is the filtering. The other two are preliminary operations that help to carry out the filtering operation under extreme conditions. frequency shifting operation helps to increase the down-sampling ratio to the maximum. Based on these three operations, the iterations of the algorithm are arranged as follows:

$$
1 q=1, \mathbf{B}_{0}=\mathbf{B}, y_{0}[n]=y[n] \text {, and } F_{s, 0}=F_{s, y} \text {. }
$$

2 In iteration $(q)$ :

- Verify if the stability criterion of the elliptic filter is satisfied over the target band $\mathbf{B}_{q}$. If so, go to the next step, otherwise skip the next step.

- Perform a filtering operation over $\mathbf{B}_{q}$ on $y_{q-1}[n]$ to get the filtered signal $y_{\mathbf{B}}[n]$, and go to step (3).

- Perform a frequency shifting operation, which consists of:

- Calculate the negative frequency shift $-\Delta f_{q}$. Apply a high-pass filter over $\left[\Delta f_{q}, \frac{F_{s, q-1}}{2}\right]$ on $y_{q-1}[n]$, and carry out frequency shifting to yield

$$
y_{\text {shift }, q}[n]=\Re\left\{e^{-2 \pi \Delta f_{q} \frac{n}{F_{s, q-1}}} \times \mathcal{H}\left\{y_{q}[n]\right\}\right\},
$$

where $\Re$ is the operator to take the real part, and $\mathcal{H}\{\cdot\}$ is the Hilbert transform.

$-\mathbf{B}_{q}=\mathbf{B}_{q-1}-\Delta f_{q}$.

- Perform a down-sampling operation.

- Calculate the down-sampling rate $D_{q}$. Apply a low-pass filter over $\left[0, \frac{F_{s, q-1}}{2 D_{q}}\right]$ on $y_{q-1}[n]$, and down-sample the signal as

$$
y_{\text {down }, q}[n]=\text { Decimate }\left\{y_{\text {shift }, q}[n] ; D_{q}\right\},
$$

- $F_{s, q}=\frac{F_{s, q-1}}{D_{q}}$

- Update the signal for the next iteration $y_{q+1}[n]=y_{\text {down }, q}[n]$. Increment the iteration index $q \leftarrow q+1$, and perform step (2) again. 
$3 F_{s}=F_{s, q}$, define the filtered signals as $y_{\mathbf{B}}[n]$.

The great advantage of the proposed method is that it is a data-driven approach. The multi-rate filter can automatically choose the optimal number of iterations and the optimal configurations of the operations.

\subsection{Time Synchronous Averaging}

The time synchronous averaging calculates a period-wise average of a periodical signal. It also helps to eliminate all of the spectral content that is incoherent with the period. A review on the available methods for time synchronous averaging is presented in [32].

Assuming that the period of the signal obtained by the multi-rate filter is sampled at $T_{y_{\mathrm{B}}}=F_{s} / f_{\Phi}$ points per period, which is often not an integer number, the signal $y_{\mathrm{B}}$ has to be a-priori resampled with an interpolation, to contain an integer number $T_{\mathbb{Z}}$ of points per period, which is a classical method [33]. The interpolated signal $y_{T_{Z}}[n]$ is further processed by

$$
\overline{y_{T_{\mathbb{Z}}}}[\tau]=\frac{\sum_{t=0}^{N_{T}-1} y_{T_{\mathbb{Z}}}\left[t T_{\mathbb{Z}}+\tau\right] \times w[t]}{\sum_{t=0}^{N_{T}-1} w[t]} .
$$

where $\tau$ is the discrete time index, $\overline{y_{T_{Z}}}[\tau]$ is the averaged signal, $N_{T}$ is the total number of periods of $\mathbf{y}_{T_{\mathbb{Z}}}$, and $w[t], t=0, \ldots, N_{T}-1$ is a window of $N_{T}$ points. To reduce the border effects of the filtering, we propose to use a Hann window.

\subsection{Demodulation Based on the Synchronous Averaged Signal}

The averaged signal $\overline{y_{T_{Z}}}[\tau]$ obtained from Equation (14) after the multi-rate filtering can be regarded as band-limited and mono-component, as it contains only one carrier and its associated sidebands. Therefore, a Hilbert transform can be applied to yield an analytical signal [34]

$$
\overline{y_{H}}[\tau]=\overline{y_{T_{Z}}}[\tau]+j \cdot \mathcal{H}\left\{\overline{y_{T_{\mathbb{Z}}}}[\tau]\right\},
$$


where $j$ is the imaginary unit, and $\mathcal{H}\{\cdot\}$ is the Hilbert transform. $\overline{y_{H}}[\tau]$ can be represented by

$$
\overline{y_{H}}[\tau]=\hat{A}[\tau] e^{j \hat{\Phi}[\tau]}
$$

where $\hat{A}[\tau]$ and $\hat{\Phi}[\tau]$ are estimations of the demodulated amplitude $A[\tau]$ and the phase $\Phi[\tau]$, respectively, defined in Equations (6) and (7), and can be calculated from the modulus and the argument of $\overline{y_{H}}[\tau]$

$$
\begin{gathered}
\hat{A}[\tau]=\left|\overline{y_{H}}[\tau]\right|, \\
\hat{\Phi}[\tau]=\arctan \left(\frac{\operatorname{imag}\left(\overline{y_{H}}[\tau]\right)}{\operatorname{real}\left(\overline{y_{H}}[\tau]\right)}\right) .
\end{gathered}
$$

The demodulated frequency $\hat{F}[\tau]$ can be obtained by differentiating $\Phi[\tau][35]$

$$
\hat{F}[\tau]=\frac{1}{2 \pi} \frac{\hat{\Phi}[\operatorname{tau}]-\hat{\Phi}[\operatorname{tau}-1]}{F_{S}} .
$$

\subsection{Features of Modulation Sidebands}

We propose to use the demodulated signals in the time domain to also compute the statistical health indicators. Table 3 shows the scalar features we

derived from the demodulated functions $\hat{A}[\tau]$ and $\hat{F}[\tau]$ as mechanical fault indicators.

A further run of AStrion $[19,21]$ on $\hat{A}[\tau]$ and $\hat{F}[\tau]$ yields the number of peaks and the energy of the harmonic series on the spectrum. These numbers contribute to two additional features for $\hat{A}[\tau]$ and $\hat{F}[\tau]$.

\section{Case Study}

This section presents the results of the above-proposed algorithms. The section starts with a description of the test rig where the vibration data are collected. The following subsections present the results of the kinematic association, the demodulation on one sample, and the final outcome of the demodulation step through the lifetime of the rolling-element bearing. Finally, a comparison with standard features, such as RMS, crest factor, kurtosis, and narrow-band RMS, is presented as a validation of the proposed method. 
Table 3: Features calculated from the demodulated amplitude $\hat{A}[\tau]$ and frequency $\hat{F}[\tau]$

\begin{tabular}{|l||c|c|}
\hline Mean & $\bar{A}=\frac{\sum_{\tau=1}^{T} \hat{A}[\tau]}{T}$ & $\bar{F}=\frac{\sum_{\tau=1}^{T} \hat{F}[\tau]}{T}$ \\
\hline Peak-to-Peak & $P P_{A}=\max \hat{A}[\tau]-\min \hat{A}[\tau]$ & $P P_{F}=\max \hat{F}[\tau]-\min \hat{F}[\tau]$ \\
\hline Kurtosis & $\operatorname{Kurt}_{A}=\frac{\left(\sum_{\tau=1}^{T} \hat{A}^{4}[\tau]\right) / T}{\left[\left(\sum_{\tau=1}^{T} \hat{A}^{2}[\tau]\right) / T\right]^{2}}$ & $\operatorname{Kurt}_{F}=\frac{\left(\sum_{\tau=1}^{T} \hat{F}^{4}[\tau]\right) / T}{\left[\left(\sum_{\tau=1}^{T} \hat{F}^{2}[\tau]\right) / T\right]^{2}}$ \\
\hline $\begin{array}{l}\text { Modulation } \\
\text { index }\end{array}$ & $M I_{A}=\frac{\max \{\hat{A}[\tau]\}-\min \{\hat{A}[\tau]\}}{\bar{A}}$ & $M I_{F}=\frac{\max \{\hat{F}[\tau]\}-\min \{\hat{F}[\tau]\}}{\bar{F}}$ \\
\hline
\end{tabular}

Table 4: Selected characteristic frequencies expressed in $H z-S S F$ of the low-speed shaft, and the main bearing related frequencies.

\begin{tabular}{|l|l|l|l|l|}
\hline SSF & BPFO & BPFI & $F T F$ & $B S F 2$ \\
\hline 0.333 & 2.543 & 3.457 & 0.140 & 1.057 \\
\hline
\end{tabular}

\subsection{Test Rig Description}

On behalf of the European Innovation Project 'KAStrion' (more details can was designed and installed at CETIM, France (http://www.cetim.fr). This test rig simulates a wind turbine drive train configuration at a smaller scale, with a $10-\mathrm{kW}$ generator. Also, hydraulic cylinders were installed to enable control of the external forces on the bearings. This provides the possibility for accelerated deterioration test allows us to observe changes in the vibrations of a new and healthy bearing, up to those of the bearing with a naturally generated fault.

Figure 2 presents the most important kinematic components of the test rig, including the motor, gearbox, generator, and the main bearing that is investigated here. The motor has the possibility of controlling the input speed as constant or variable in the tests conducted. The speed ratio of the low-speed shaft over the high-speed shaft is 1:100.75. 


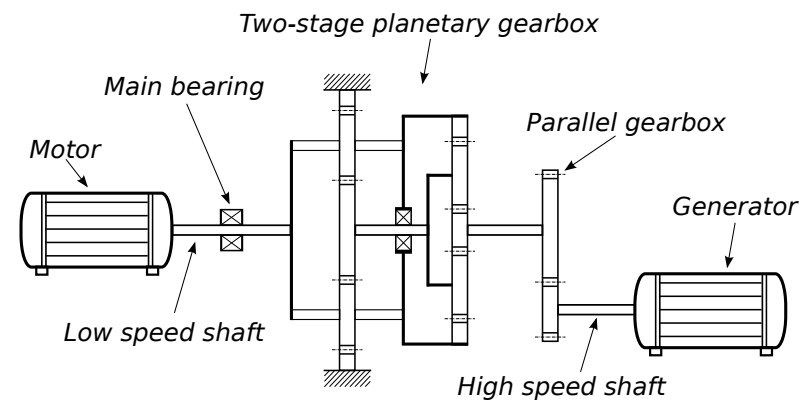

Figure 2: Schematic representation of the test rig designed as a wind turbine.

The relevant components with their theoretical characteristic fault frequencies are given in Table 4, which gives the low-speed shaft frequency $S S F$ and see Equation 1) computed in the association process. It is common that for realworld signals large numbers of harmonic and sideband series are left without association, and this step serves also for the selection of the more interesting 
Table 5: Example of a harmonic and modulation sideband series association on the test rig data.

\begin{tabular}{|c|c|c|c|c|c|c|}
\hline Type & $\begin{array}{c}\text { Frequency } \\
(\mathbf{H z})\end{array}$ & $\begin{array}{c}\text { Carrier } \\
\text { frequency } \\
(\mathbf{H z})\end{array}$ & $\begin{array}{c}\text { Modulation } \\
\text { frequency } \\
(\mathbf{H z})\end{array}$ & $\begin{array}{c}\text { Associated } \\
\text { component } \\
\text { name }\end{array}$ & $\begin{array}{c}\text { Theoretical } \\
\text { frequency } \\
(\mathbf{H z})\end{array}$ & RFD $_{i}$ \\
\hline Harmonic & 2.565 & - & - & $\begin{array}{c}\text { Main Bearing } \\
\text { BPFO }\end{array}$ & 2.543 & 0.845 \\
\hline Harmonic & 3.435 & - & - & $\begin{array}{c}\text { Main Bearing } \\
\text { BPFI }\end{array}$ & 3.457 & -0.623 \\
\hline Sidebands & - & 3.435 & 1.003 & Planetary Gear & 1.000 & 0.271 \\
\hline Sidebands & - & 3.435 & 0.334 & Low-Speed Shaft & 0.333 & 0.271 \\
\hline Sidebands & - & 6.870 & 0.333 & Low-Speed Shaft & 0.333 & -0.034 \\
\hline
\end{tabular}

series for further analyses. As described in section 3, the demodulation is per315 formed according to the results of the characteristic frequency association. All of the detected sidebands are demodulated automatically by the method proposed in section 3. The following subsections show only the results associated with the modulation of the main bearing characteristic frequencies by the low-speed shaft, where the main bearing was mounted.

\subsection{Feature Set of the Sideband Demodulation}

Over the sidebands with carrier frequency $f_{0}=3.435 \mathrm{~Hz}$, the modulation frequency $f_{\Phi}=0.334 \mathrm{~Hz}$, the highest sideband on the left-hand side $K_{\text {inf }}=-4$, and the highest sideband on the right-hand side $K_{\text {sup }}=6$ identified by the harmonic and sideband detection algorithm [21], the kinematic association algorithm proposed in this paper deduces that the frequency bandwidth $\mathbf{B}=[2.099 \mathrm{~Hz}, 5.439 \mathrm{~Hz}]$ contains the sidebands corresponding to the lowspeed shaft frequency $0.333 \mathrm{~Hz}$ around the carrier frequency associated with the main bearing BPFI $3.457 \mathrm{~Hz}$. Thus, the frequency bandwidth $\mathbf{B}$ is chosen as the filter bandwidth for the multi-rate filter. This frequency bandwidth is unsymmetrical around the carrier frequency and is extremely narrow (the normalized bandwidth $\overline{\Delta B}=0.017$ ), which implies a very difficult filtering step. As presented in section 3.2, the multi-rate filter accomplished the filtering in 
Table 6: Features calculated from the demodulated amplitude and frequency that describe a vibration signal that corresponds to the main bearing BPFI frequency modulated by the low-speed shaft.

\begin{tabular}{|c|c|c|c|}
\hline Mean $\bar{A}$ & Peak-to-Peak $P P_{A}$ & Kurtosis ur $_{A}$ & Modulation index $M I_{A}$ \\
\hline 0.0013 & 0.0018 & 1.9134 & 0.7138 \\
\hline \hline Mean $\bar{F}$ & Peak-to-Peak $P P_{F}$ & Kurtosis Kur $_{F}$ & Modulation index $M I_{F}$ \\
\hline 3.435 & 2.725 & 6.773 & 0.397 \\
\hline
\end{tabular}

four iterations, which included the automatic selection of the frequency shift and the signal decimation. Finally, the down-sampled signal is sampled with a frequency of $13.58 \mathrm{~Hz}$. Figure 3 presents the results of the multi-rate filtering.

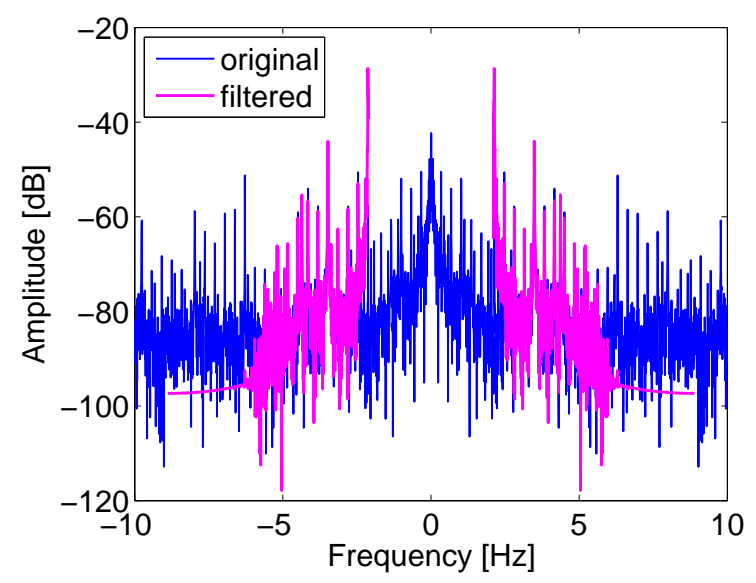

Figure 3: Spectrum of the original signal and the signal filtered by the proposed multi-rate filter over the bandwidth $\mathbf{B}=[2.099 \mathrm{~Hz}, 5.439 \mathrm{~Hz}]$ - zoom of the interesting range.

Following the synchronous averaging carried out as specified in section 3.3 and the demodulation as in section 3.4, the features calculated from the demodulated amplitude and frequency as defined in section 3.5 are listed in Table 6 . 


\subsection{Feature Tracking over a Sequence of Signals}

The above-presented feature calculation was applied for 16 vibration measurements that covered the time span of the main bearing degradation test, from the new main bearing condition up to its spread fault in the $190^{\text {th }}$ hour.

Figure 4 presents the selected results of the AStrion methodology. Figure 4a shows the changes in the energy of the harmonic series associated with the main bearing BPFI. The details of the computing of this indicator can be found in [21], and here it serves to demonstrate the robustness of the algorithm. There are two features of the proposed sideband demodulation method shown in Figure 4b, c, which show the average $\bar{A}$ and the modulation index $M I_{A}$, respectively, of the estimated amplitude modulation function (see Table 3). Both of these indicators

350 are non-zero when the modulation sidebands are detected. Among all of the modulation sidebands revealed, the only ones shown are the series with the carrier frequency corresponding to the fundamental frequency, the $2^{\text {nd }}$, and the $3^{\text {rd }}$ harmonics of the main bearing BPFI, and the modulation frequency linked to the low-speed shaft frequency.

It is important to note that the value is computed only in the case of the detection of the investigated frequency. The first available sample of the data is acquired in the $44^{\text {th }}$ hour, but the first non-zero value of the indicators presented in Figure 4a corresponds to the $129^{\text {th }}$ hour. Figure $4 \mathrm{~b}$, c has the first values in the $134^{\text {th }}$ hour. This is because from the $129^{\text {th }}$ hour it is possible to detect harmonic series associated with the main bearing BPFI, whereas from the $134^{\text {th }}$ hour, detection of the modulation associated with the low-speed shaft is possible. The $129^{\text {th }}$ hour is considered as the first warning of the investigated fault, and the $134^{\text {th }}$ hour is considered as the time of the fault detection by the method presented.

365 The average $\bar{A}$ presented in Figure $4 \mathrm{~b}$ shows an abrupt increase at the beginning that is the largest for the fundamental frequency of the main bearing BPFI. This rapid jump corresponds to the increasing dimension of the fault on the inner ring of the rolling-element bearing. The impact caused by the rolling elements passing the local fault on the inner race of the main bearing gener- 


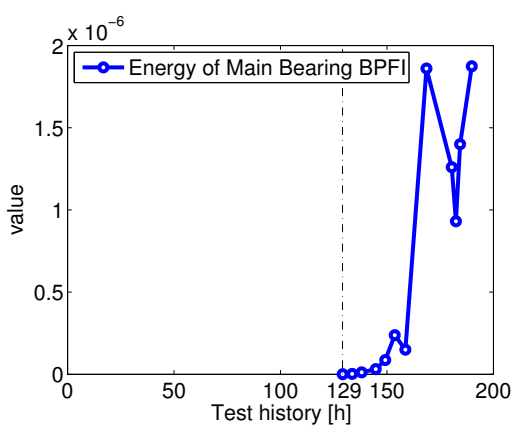

(a) Harmonic Series Energy

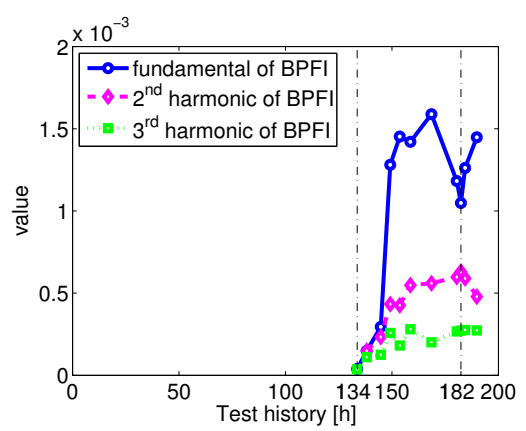

(b) $\bar{A}$

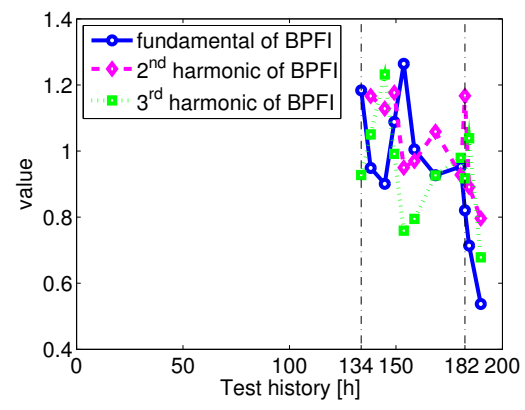

(c) $M I_{A}$

Figure 4: Evolution of (a) the energy of the harmonic series associated with the main bearing BPFI, (b) the mean value of the amplitude demodulation function $\bar{A}$, and (c) the modulation index $M I_{A}$ over the detected modulation around the fundamental frequency, $2^{\text {nd }}$ harmonic, and $3^{\text {rd }}$ harmonic of the main bearing BPFI through the lifetime of the main bearing tested in the KAStrion test rig.

ates more energy from sample to sample. Most of this energy is present in the fundamental frequency, and its harmonics have less and less energy, which can also be observed in Figure 4b. After the $149^{\text {th }}$ hour, the value of the indicator fluctuates, and it changes more abruptly in the $182^{\text {nd }}$ hour.

The modulation index $M I_{A}$ in Figure 4c shows a steady trend from the beginning of the sideband detection. The values of $M I_{A}$ are on a similar level for the fundamental frequency, $2^{\text {nd }}$, and $3^{\text {rd }}$ harmonics of main bearing BPFI. In the $182^{\text {nd }}$ hour, the indicators start to drop. We interpret this as a change in 
the fault nature of the bearing under investigation. The weakening modulation corresponds to a more and more distributed fault, as the impacts caused by the rolling elements in motion are no longer as periodic as in the case of a local fault. The fault becomes spread over the inner race of the main bearing.

Through simultaneous interpretation of the $\bar{A}$ and $M I_{A}$ indicators, it is possible to trace the nature of the fault. This is confirmed by visual inspection of the main bearing after the end of the test, where the entire inner race of the investigated bearing was covered with flaking after it was disassembled from the test rig. Figure 5 shows a photo of the bearing at the end of the deterioration test.

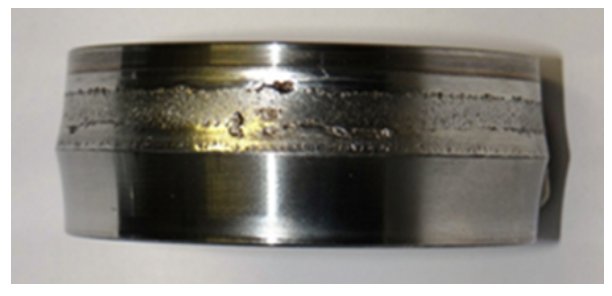

Figure 5: Flaking over the entire inner race of the faulty bearing.

Moreover, AStrion does all of the computation fully automatically, and there is no need for any adjustment of the parameters by the user. All of the parameters are estimated in the investigated signal, including the range of the sidebands for the demodulation. This approach gives a well-designed and robust tool, which can also be used by a non-expert in signal-processing techniques.

\subsection{Comparison to Established CMS Techniques}

Figure 6 shows the results of the well-known techniques used in CMS applied to the same vibration signals. The RMS and kurtosis in Figure 6a, c, respectively, are not sufficient for clear detection of the fault. Among the simplest fault indicators, only the crest factor, as presented in Figure $6 \mathrm{~b}$, enables the detection of the fault in the $169^{\text {th }}$ hour, which is much later than the proposed method. Furthermore, this indicator cannot identify the faulty bearing. 


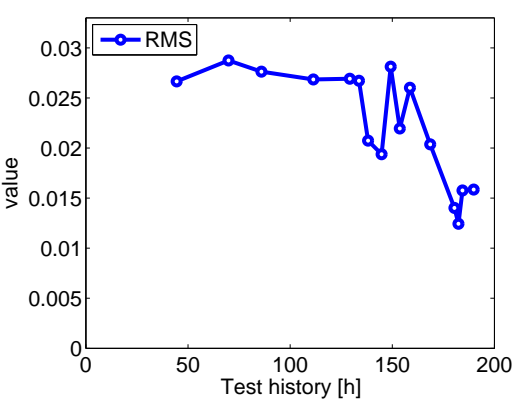

(a) RMS

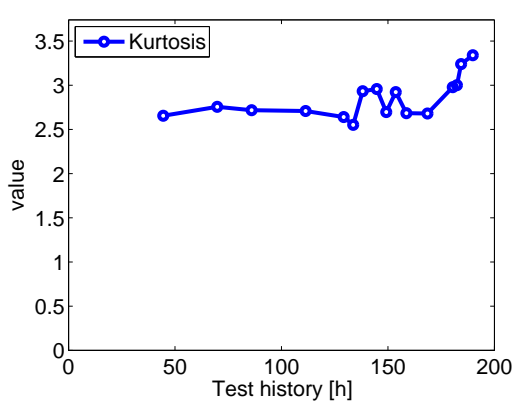

(c) Kurtosis

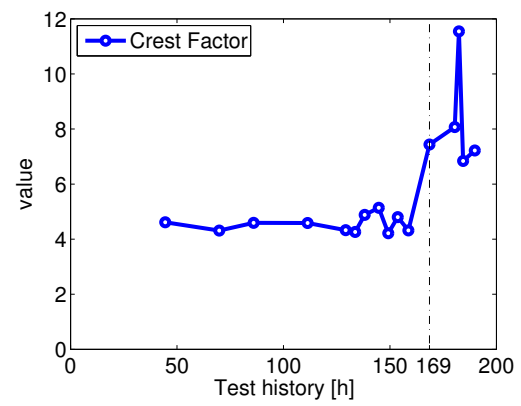

(b) Crest Factor

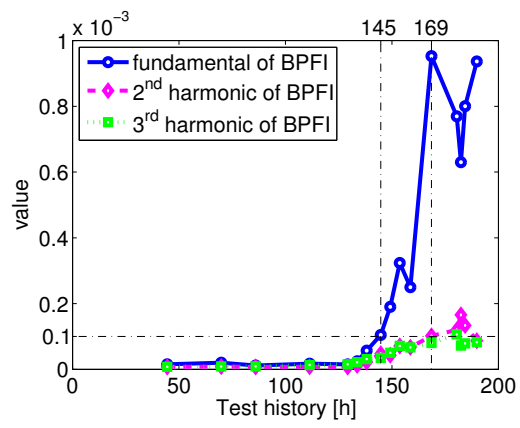

(d) Narrow-Band RMS

Figure 6: Evolution of the (a) RMS, (b) crest factor, and (c) kurtosis values computed for the full-length signal, and the (d) narrow-band RMS with $3 \%$ bandwidth of the fundamental frequency, $2^{\text {nd }}$ harmonic, and $3^{\text {rd }}$ harmonic of the BPFI of the main bearing through the main bearing lifetime.

The narrow-band RMS is the only indicator where the performance is comparable with the AStrion methodology in the investigated case. Figure 6d shows the narrow-band RMS computed around the fundamental frequency, $2^{\text {nd }}$ harmonic, and $3^{\text {rd }}$ harmonic of the main bearing BPFI. The bandwidth of this feature is $3 \%$ of the investigated frequency, which is based on engineering practice for CMS. The threshold for fault detection is selected as 0.0001. The disadvantage of this method is that the threshold depends on the user's experience and on other human factors. With this selection, the detection based on narrowband RMS of the fundamental frequency of the main bearing BPFI is in the 
$145^{t h}$ hour, which is shown by the vertical dash-dot line in Figure $6 \mathrm{~d}$. This is late detection in comparison with the proposed method, where the $134^{\text {th }}$ hour is achieved. The narrow-band RMS of the $2^{\text {nd }}$ harmonic can detect the fault in the $169^{\text {th }}$ hour. The narrow-band RMS of the $3^{\text {rd }}$ harmonic also crosses the threshold, but only for one sample in the $180^{\text {th }}$ hour, and it then drops below the threshold. This behavior might be seen as a false alarm. Additionally, the narrow-band RMS gives no clue about the nature of the fault.

Moreover, it is worth mentioning that in the investigated case, a lot of wellestablished methods of rolling bearing diagnosis fail because of the low rotational speed of the main bearing. There is no effect of exciting the resonance frequencies by a faulty bearing, so methods based on envelope analysis or cyclostation${ }_{420}$ ary analysis of the $2^{\text {nd }}$ order cannot detect the investigated fault. Examples of these methods are presented in [24, 22].

\section{Conclusions}

This paper focuses on signal-processing techniques for automatic and datadriven CMS. The two proposed methods are automatic, and are based on the results of harmonic and sideband series detection that is performed without any a-priori information on the signal. The first proposed algorithm is a characteristic frequency association method, as a two-step approach that takes into account the possible slip of the rolling-element bearings. The second proposed method is an all-sideband demodulation algorithm.

430 The results presented here show that the kinematic frequency association algorithm can carry out efficient research of the characteristic frequencies of the mechanical components with the help of the kinematic configuration of the machine. The real-world results show the applicability of the sideband demodulation algorithm to very difficult situations where the filter band is asymmetric

435 and extremely narrow. This allows for deeper insight into the modulations created by the fault, and calculates the features relative to the evolution of the fault. The proposed method can detect a rolling-element bearing BPFI fault in 
an automatic way, and using a data-driven approach. The detection is very early compared with the well-established techniques presented in section 4.5. Moreover, the proposed features characterise the nature of the investigated fault.

The integration of the results obtained in the surveillance of the system is the next step [36]. As the whole algorithm is applied in a fully automatic way, this makes the proposed approach a good solution for CMS application. In further studies, and in the context of the two European projects 'KAStrion'

445 and 'SUPREME', integration of these proposed algorithms into a commercial wind turbine CMS is scheduled.

Another interesting task in the context of the Innovation Project KAStrion, which aims to develop an advanced CMS dedicated to offshore wind turbine farms, and in the context of the presented work is adding an influence of the load in the investigated system. As [37] points out the load susceptibility characteristics is required in the machine condition monitoring which works in the non-stationary operation conditions. In order to investigate this aspect it would be interesting to demonstrate the relation between the proposed statistical features and condition parameters, in the similar way as proposed for popular health indicators in [38]. These issue will be addressed in a future work.

\section{Acknowledgement}

All of the data were provided by the Center for Technology and Innovation Management (CETIM), within the 'KAStrion' project, and thanks to Sophie Sieg-Zieba. The authors would also like to thank VALEMO for their involvement 460 in the Innovation Project 'KAStrion'.

The Innovation Project 'KAStrion' was supported by KIC InnoEnergy, which is a company supported by the European Institute of Innovation and Technology (EIT), which has the mission of delivering commercial products and services, new businesses, innovators and entrepreneurs in the field of sustainable

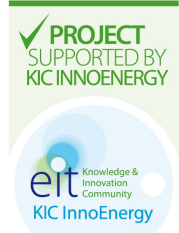


energy through the integration of higher education, research, entrepreneurs and business companies.

This study was supported by French Research National Agency (ANR) through the EITE program (project KAStrion ANR-12-EITE-0002-01).

\section{References}

[1] F. P. García Márquez, A. M. Tobias, J. M. Pinar Pérez, M. Papaelias, Condition monitoring of wind turbines: Techniques and methods, Renewable Energy 46 (2012) 169-178. doi:10.1016/j.renene.2012.03.003.

${ }_{475}[2]$ W. Liu, B. Tang, J. Han, X. Lu, N. Hu, Z. He, The structure healthy condition monitoring and fault diagnosis methods in wind turbines: A review, Renewable and Sustainable Energy Reviews 44 (2015) 466-472. doi:10.1016/j.rser.2014.12.005.

[3] K. K. Agrawal, P. G. N. Pandey, K. Chandrasekaran, Analysis of the Condition Based Monitoring System for Heavy Industrial Machineries, Computational Intelligence and Computing Research (ICCIC), 2013 IEEE International Conference on. doi:10.1109/ICCIC.2013.6724183.

[4] E. Egusquiza, C. Valero, D. Valentin, A. Presas, C. G. Rodriguez, Condition monitoring of pump-turbines. New challenges, Measurement 67 (2015) 151-163. doi:10.1016/j.measurement.2015.01.004.

[5] D. Banjevic, D. Lin, A. K. Jardine, A review on machinery diagnostics and prognostics implementing condition-based maintenance, Mechanical Systems and Signal Processing 20 (7) (2006) 1483-1510. doi:10.1016/ j.ymssp. 2005.09.012.

490 [6] W. Bartelmus, R. Zimroz, Vibration condition monitoring of planetary gearbox under varying external load, Mechanical Systems and Signal Processing 23 (1) (2009) 246-257. doi:10.1016/j .ymssp. 2008.03.016. 
[7] R. F. Mesquita Brandão, J. A. Beleza Carvalho, F. P. MacIel Barbosa, Forecast of faults in a wind turbine gearbox, Proceedings of 9th International Conference, ELEKTRO 2012 (2012) 170-173. doi:10.1109/ ELEKTRO . 2012.6225632.

[8] W. Yang, P. J. Tavner, R. Court, An online technique for condition monitoring the induction generators used in wind and marine turbines, Mechanical Systems and Signal Processing 38 (1) (2013) 103-112. doi: $10.1016 / j \cdot y m s s p .2012 .03 .002$.

[9] P. Tchakoua, R. Wamkeue, T. A. Tameghe, G. Ekemb, A review of concepts and methods for wind turbines condition monitoring, 2013 World Congress on Computer and Information Technology (WCCIT) 2 (1) (2013) 1-9. doi: 10.1109/WCCIT. 2013.6618706.

[10] T. Barszcz, M. Strączkiewicz, Novel Intuitive Hierarchical Structure for Condition Monitoring System of Wind Turbines, Diagnostyka 14 (3) (2013) 53-60.

[11] P. J. Tavner, D. Zappal, S. Sheng, C. J. Crabtree, Side-band algorithm for automatic wind turbine gearbox fault detection and diagnosis, IET Renewable Power Generation 8 (4) (2014) 380-389. doi:10.1049/iet-rpg. 2013.0177.

[12] P. D. McFadden, Detecting fatigue cracks in gears by amplitude and phase demodulation of the meshing vibration, Journal of Vibration, Acoustics, Stress, and Reliability in Design 108 (1986) 165-170.

515 [13] R. B. Randall, Developments in digital analysis techniques for diagnostics of bearings and gears, in: Fifth International Congress on Sound and Vibration, Adelaide, South Australia, 1997.

[14] A. R. Mohanty, C. Kar, Fault Detection in a Multistage Gearbox by Demodulation of Motor Current Waveform, IEEE Transactions on Industrial Electronics 53 (4) (2006) 1285-1297. doi:10.1109/TIE.2006.878303. 
[15] F. Combet, L. Gelman, Novel adaptation of the demodulation technology for gear damage detection to variable amplitudes of mesh harmonics, Mechanical Systems and Signal Processing 25 (3) (2011) 839-845. doi:10.1016/j.ymssp.2010.07.008.

525 [16] T. Barszcz, A. Jabłoński, Selected methods of finding optimal center frequency for amplitude demodulation of vibration signals, Diagnostyka 2 (54) (2010) 2008-2011.

[17] P. Borghesani, R. Ricci, S. Chatterton, P. Pennacchi, A new procedure for using envelope analysis for rolling element bearing diagnostics in variable operating conditions, Mechanical Systems and Signal Processing 38 (1) (2013) 23-35. doi:10.1016/j.ymssp.2012.09.014.

[18] N. Martin, C. Mailhes, A non-stationary index resulting from time and frequency domains, in: Sixth International Conference on Condition Monitoring and Machinery Failure Prevention Technologies. CM 2009 and MFPT $535 \quad 2009$, Dublin, Ireland, 2009.

[19] C. Mailhes, N. Martin, K. Sahli, G. Lejeune, A spectral identity card, in: EUropean SIgnal Processing Conference, EUSIPCO 06, Florence, Italy, 2006.

[20] C. Mailhes, N. Martin, K. Sahli, G. Lejeune, Condition monitoring using automatic spectral analysis, in: Special session on "Condition Monitoring of Machinery", Third European Workshop on Structural Health Monitoring, Structural Health Monitoring 2006, Granada, Spain, 2006, pp. 1316-1323.

[21] T. Gerber, N. Martin, C. Mailhes, Identification of harmonics and sidebands in a finite set of spectral components, in: Tenth International Con545 ference on Condition Monitoring and Machinery Failure Prevention Technologies. CM 2013 and MFPT 2013, Kraków, Poland, 2013. 
[22] G. Dong, J. Chen, Study on cyclic energy indicator for degradation assessment of rolling element bearings, Journal of Vibration and Control 17 (12) (2010) 1805-1816. doi:10.1177/1077546310362860.

[23] R. B. Randall, Vibration-based Condition Monitoring, John Wiley \& Sons, Ltd, Chichester, UK, 2011. doi:10.1002/9780470977668.

[24] R. B. Randall, J. Antoni, Rolling element bearing diagnostics - A tutorial, Mechanical Systems and Signal Processing 25 (2) (2011) 485-520. doi: $10.1016 / j \cdot y m s s p .2010 .07 .017$.

555 [25] K. Fyfe, E. Munck, Analysis of computed order tracking, Mechanical Systems and Signal Processing 11 (2) (1997) 187-205.

[26] M. Firla, Z.-Y. Li, N. Martin, T. Barszcz, Automatic and Full-band Demodulation for Fault Detection - Validation on a Wind Turbine Test Rig, in: 4th International Conference on Condition Monitoring of Machinery in Non-Stationary Operations (CMMNO'2014), France, Lyon, 2014.

[27] T. Barszcz, A. Jabłoński, A novel method for the optimal band selection for vibration signal demodulation and comparison with the Kurtogram, Mechanical Systems and Signal Processing 25 (1) (2011) 431-451. doi: $10.1016 / j \cdot y m s s p \cdot 2010.05 .018$.

[28] J. Obuchowski, A. Wyłomańska, R. Zimroz, Selection of informative frequency band in local damage detection in rotating machinery, Mechanical Systems and Signal Processing 48 (1-2) (2014) 138-152. doi:10.1016/j . ymssp. 2014.03.011.

[29] C. Pachaud, T. Gerber, M. Firla, N. Martin, C. Mailhes, Consequences of non-respect of the Bedrosian theorem when demodulating, in: Tenth International Conference on Condition Monitoring and Machinery Failure Prevention Technologies. CM 2013 and MFPT 2013, Kraków, Poland, 2013. 
[30] R. Meyer, C. S. Burrus, Design and implementation of multirate digital filters, Acoustics, Speech and Signal Processing, IEEE Transactions on 24 (1) (1976) 55-58.

[31] J. Carson, Notes on the theory of modulation, Proc. IRE 10 (1) (1922) $57-64$.

[32] E. Bechhoefer, M. Kingsley, A review of time synchronous average algorithms, Annual conference of the prognostics and health management society (2009) 1-10.

[33] P. McFadden, A revised model for the extraction of periodic waveforms by time domain averaging, Mechanical Systems and Signal Processing 1 (1) (1987) 83-95. doi:10.1016/0888-3270(87)90085-9.

[34] D. Ho, R. B. Randall, Optimisation of Bearing Diagnostic Techniques Using Simulated and Actual Bearing Fault Signals, Mechanical Systems and Signal Processing 14 (5) (2000) 763-788. doi:10.1006/mssp.2000.1304.

[35] B. Boashash, Time-Frequency Signal Analysis and Processing - A Comprehensive Reference, Elsevier Science, Oxford, UK, 2003.

[36] T. Gerber, N. Martin, C. Mailhes, Time-Frequency Tracking of Spectral Structures Estimated by a Data-Driven Method, IEEE Transactions on Industrial Electronics 62 (10) (2015) 6616-6626. doi:10.1109/TIE. 2015. 2458781.

[37] W. Bartelmus, Editorial statement, Mechanical Systems and Signal Processing 38 (1) (2013) 1-4, Condition monitoring of machines in nonstationary operations. doi:http://dx.doi.org/10.1016/j.ymssp. 2013. 04.003.

[38] R. Zimroz, W. Bartelmus, T. Barszcz, J. Urbanek, Diagnostics of bearings in presence of strong operating conditions non-stationarity - a procedure of load-dependent features processing with application to wind turbine 


\section{Appendix A. Down-sampling Rates and Frequency Shift Steps}

The distribution of the down-sampling rates in all of the iterations is an important configuration of this method, and it depends first on the final sampling frequency $F_{s}=C \cdot \Delta B$, where $C$ is a factor between 4 and 10 that ensures a wide filter band and a high sampling frequency at the same time. The overall down-sampling rate $D$ is factorized by $D=2^{L^{(2)}} 3^{L^{(3)}}$ by

$$
\begin{array}{ll} 
& \left\{L^{(2)}, L^{(3)}\right\}=\underset{\left\{l^{(2)}, l^{(3)}\right\} \in \mathbb{Z}^{2}}{\arg \max }\left\{\frac{F_{s_{y}}}{F_{s}}-2^{l^{(2)}} 3^{l^{(3)}}\right\} \\
\text { subject to } & \frac{F_{s_{y}}}{F_{s}}-2^{l^{(2)}} 3^{l^{(3)}} \geq 0
\end{array}
$$

The factors 2 and 3 are alternatively arranged in a vector $\mathbf{d}$

$$
\mathbf{d}=\left\{d_{l}\right\}_{L^{(2)}+L^{(3)}}=[2,3,2,3, \ldots] .
$$

$L_{q}^{(2)}$ factors of 2 and $L_{q}^{(3)}$ factors of 3 will be taken from $\mathbf{d}$ in each downsampling operation, to form the down-sampling factor $D_{q}$. At the end of all of the iterations, $\mathbf{d}$ will be empty $\mathbf{d}=\emptyset$. Therefore, the total number of iterations $N_{q}$ cannot exceed $\left(L^{(2)}+L^{(3)}\right)$.

In each iteration $(q)$ in step (2.4.1), the algorithm calculates the downsampling factor $D_{q}$ by selecting $L_{q}$ elements from $\mathbf{d}$ according to $D_{q}=\prod_{l=1}^{L_{q}+1} d_{l}$, where $L_{q}$ defines the narrowest band $\left[0,\left(F_{s, q-1}\right) /\left(2 D_{q}\right)\right]$ where a low-passe Butterworth filter satisfies the stability criterion. $\mathbf{d}$ is then updated by removing the first $L_{q}$ factors $\mathbf{d} \leftarrow \mathbf{d} /\left[d_{1}, \ldots, d_{L_{q}}\right]$. If $D_{q}=1$, the down-sampling operation will simply be skipped.

The operations aim to down-sample the signal to a sufficiently low sampling frequency to achieve the biggest filter bandwidth $\Delta B$. Hence, the frequency

${ }_{615}$ shifting operations will carry the filter band $\mathbf{B}$ to $\left(\mathbf{B}-f_{\text {sup }}\right)$ where the lower frequency boundary is 0 . The total frequency shift is therefore $-f_{\text {sup }}$ and is divided evenly into $M$ smaller portions. In each iteration $(q)$, the algorithm 
takes $M_{q}$ portions to shift the frequency contents of the signal by $-\Delta f_{q}=$ $-\frac{M_{q}}{M} f_{\text {sup }}$.

In each iteration $(q)$, step (2.3.1) calculates $M_{q}$ by finding the narrowest band $\left[\frac{M_{q}+1}{M} f_{\text {sup }},\left(F_{s, q-1}\right) / 2\right]$ where a high-pass Butterworth filter satisfies the stability criterion. $\Delta f$ is then updated by

$$
\Delta f \leftarrow \Delta f-\Delta f_{q}
$$

620 If the above process returns $\Delta f_{q}=0$, the frequency shifting operation will be skipped. 